\title{
ON A QUESTION RAISED BY GARRETT BIRKHOFF
}

\author{
E. T. PARKER
}

Birkhoff ${ }^{1}$ proposes the problem: Determine the largest integer, $k$, such that each group whose order is the product of $k$ primes (not necessarily distinct) has subgroup lattice of length $k$. The condition is satisfied by a group, $G$, if and only if $G$ contains a sequence of subgroups,

$$
G, G_{1}, G_{2}, \cdots, G_{k}=\text { identity, }
$$

each a subgroup of prime index in the preceding. He outlines a proof ${ }^{2}$ that each group with $k \leqq 4$ has this property. An example will now be given of a group, with $k=5$, which does not satisfy the condition, since it has no subgroup of prime index.

There exists a simple group, ${ }^{3} G$, of order $2^{2} \cdot 3 \cdot 7 \cdot 13$.

$G$ has no subgroup of index 2, for such a subgroup would be normal ${ }^{4}$ in $G$, in contradiction to the fact that $G$ is simple.

$G$ has $2 \cdot 3 \cdot 13$ Sylow subgroups ${ }^{5}$ of order 7 , for no other divisor $(>1)$ of $2^{2} \cdot 3 \cdot 13$ is $\equiv 1(\bmod 7)$; and the normalizer ${ }^{5}$ in $G$ of each Sylow subgroup of order 7 is of order 2.7. A subgroup, $H$, of index 3 or 13 in $G$ could have only one Sylow subgroup of order 7, necessarily normal in $H$ of order respectively $2^{2} \cdot 7 \cdot 13$ or $2^{2} \cdot 3 \cdot 7$, both of which are larger than $2 \cdot 7$. Hence, $G$ has no subgroup of index 3 or 13 .

$G$ has 2.7 Sylow subgroups of order 13 ; the normalizer in $G$ of each is of order $2 \cdot 3 \cdot 13$. A subgroup, $M$, of index 7 in $G$ could have only one Sylow subgroup of order 13, normal in $M$ of order $2^{2} \cdot 3 \cdot 13$ $>2 \cdot 3 \cdot 13$. And $G$ has no subgroup of index 7 .

In the direct product of $G$ above and any solvable group, each subgroup of prime index must contain $G$. Hence, for each integer $n \geqq 5$, there exists a group with $n$ factors in its order not satisfying the condition.

UNIVERSITY OF TEXAS

Received by the editors December 26, 1950.

1 Birkhoff, Laitice theory, Amer. Math. Soc. Colloquium Publications, vol. 25, rev. ed., Problem 39, p. 99.

2 Ibid. p. 98.

${ }^{3}$ Burnside, Theory of groups of finite order, Cambridge University Press, 1897, pp. $338,367$.

${ }^{4}$ Speiser, Die Theorie der Gruppen von endlicher Ordnung, New York, Dover, Theorem 14, p. 30.

5 Ibid. Theorem 74, p. 67. 